\title{
Telmisartan alleviates collagen type III glomerulopathy: A case report with literature review
}

\author{
QIAOYAN GUO, LIHUA LIU, PING NIE and PING LUO \\ Department of Nephrology, The Second Hospital of Jilin University, Changchun, Jilin 130041, P.R. China
}

Received December 15, 2019; Accepted July 14, 2020

DOI: $10.3892 /$ etm.2020.9269

\begin{abstract}
Collagen type III is commonly detected in the renal interstitium and vasculature; however, it is absent in healthy glomeruli. Deposition of collagen type III in the glomerular mesangium and capillary basement membranes may arise in two rare diseases, namely collagen type III glomerulopathy (CG) and nail patella syndrome. $\mathrm{CG}$ is a rare glomerular disease with no specific treatment, although supportive measures for control of hypertension and edema may help to relieve symptoms. With progression to end-stage renal disease, patients with CG may come to require dialysis and/or renal transplantation. The present study reported on a 59-year-old male who was diagnosed with CG nephrotic syndrome by immunohistochemical and electron microscopic examination of biopsy material. To the best of our knowledge, this is the first case reported in northeastern China. The angiotensin II blocker telmisartan was successfully used to alleviate renal symptoms and a literature review was performed. The present case supports the use of telmisartan as a first choice of treatment for $\mathrm{CG}$.
\end{abstract}

\section{Introduction}

Collagen type III glomerulopathy (CG) is an idiopathic glomerular disease, characterized by massive accumulation of collagen type III within the glomerular mesangial regions and basement membrane. This rare renal disease has been previously reported in Japan and occasionally in China (1). CG may be classified into two subtypes, designated as collagenofibrotic glomerulopathy and nail patella syndrome (NPS), according to the location of collagen deposition. In CG, deposits containing type III collagen fibrils are mainly located in the subendothelium and mesangium, while those in NPS are located at the basement membrane. Furthermore, the clinical symptoms

Correspondence to: Dr Ping Luo, Department of Nephrology, The Second Hospital of Jilin University, 218 Ziqiang Street, Changchun, Jilin 130041, P.R. China

E-mail: luopingjen@163.com

Key words: telmisartan, collagen type III glomerulopathy, nephrotic syndrome, immune histochemistry, electron microscopy of CG are confined to the kidney, while patients with NPS usually have a range of extra-renal manifestations (2). CG is a rare renal disease that may occur at any age in humans and animals. Although its pathogenesis remains elusive, cases with childhood onset have certain genetic components, whereas males and females are equally likely to be affected (3).

The clinical manifestations of CG are not sufficiently specific for diagnosis uninformed by pathological findings. The major clinical manifestations of $\mathrm{CG}$ are proteinuria, hypertension and subsequent slowly progressive chronic kidney disease $(1,4,5)$. Electron microscopic examination of glomerular basement membrane (GBM) biopsy specimens has revealed massive deposition of banded type III collagen fibrils (diameter, 50-60 nM) (2). In settings such as developing countries without ready access to electron microscopy, immunohistochemical (IHC) examination may be useful. Since its etiology and pathogenesis remain unknown, there are no specific treatments for $\mathrm{CG}$, which has a poor prognosis and frequently leads to end-stage kidney failure calling for renal transplant (6).

Treatment with angiotensin II receptor blockers, including telmisartan, may decrease the glomerular pressure and a previous report indicated that telmisartan is able to attenuate kidney cell apoptosis and autophagy-associated protein expression in kidney diseases (7). Furthermore, telmisartan has anti-fibrotic effects in human mesangial cells and decreased the expression levels of the peroxisome transcription factor peroxisome proliferator-activated receptor (PPAR) $\gamma$ and the L-arginine-dependent neuronal nitric oxide synthase (nNOS) to delay the progression of renal failure (8-11). To the best of our knowledge, the present study was the first to report a case of nephrotic syndrome (NS) with CG in northeastern China. Diagnosis was confirmed by IHC and electron microscopic examination of a biopsy specimen. This patient was followed up for 3 years of therapy with telmisartan, which effectively relieved the clinical symptoms and delayed the progression and loss of renal function. From this experience and a literature search, a mini-review of CG was derived, aiming to make clinicians aware of this condition and telmisartan as an efficient treatment modality.

\section{Case report}

A 59-year-old male patient was admitted to the Second Hospital of Jilin University (Changchun, China) for 
the treatment of intermittent edema without any other complaints in May 2016. The patient had suffered from intermittent edema of the lower limbs for one month. The patient did not report other history of disease or heredopathia. On examination, the patient was revealed to be hypertensive $(150 / 95 \mathrm{mmHg})$, edematous and to have albuminuria; however, the systemic examination was otherwise unremarkable. The laboratory data indicated the following levels: Proteinuria, 3+; serum total protein, $55.3 \mathrm{~g} / \mathrm{l}$; blood albumin, $27.1 \mathrm{~g} / \mathrm{l}$; blood urea nitrogen, $8.97 \mathrm{mM}$; blood uric acid, $597 \mu \mathrm{M}$; blood creatinine, $1.233 \mathrm{mg} / \mathrm{dl}$; blood triglyceride, $2.64 \mathrm{mM}$; blood cholesterol, $6.81 \mathrm{mM}$; and 24-h urine protein output, $5.44 \mathrm{~g}$, which were higher compared with normal levels. The patient's clinical and laboratory data at initial presentation are presented in Table I. According to the medical history and laboratory findings, the patient was first diagnosed with NS and consequently, renal biopsy was performed. The patient signed an informed consent agreement prior to biopsy that allowed the use of the clinical and pathological data for scientific research. Institutional Review Board approval (approval no. 2018198) was provided prior to study onset.

Immunofluorescence (IF) microscopy of the biopsy specimen revealed $\operatorname{IgA}(-), \operatorname{Ig} M(-), \operatorname{IgG}(-)$, complement (C)3(-), C4(-), C1q(-), fibronectin(-), $\kappa(-)$ and $\lambda(-)$. Under light microscopy, the sample contained 33 complete glomeruli, most of which were lobulated in shape, with occasional enlargement. Glomerular mesangial cells exhibited mildly or moderately diffuse proliferation along with endothelial cell proliferation. Light periodic acid Schiff (PAS) polysaccharide staining was detected in the mesangium and capillary loops. Renal tubular epithelial cells exhibited vacuolar degeneration and atrophy, and protein casts. Focal fibrosis and infiltration of inflammatory cells were observed in the renal interstitium. Part of the arteriole wall was slightly thickened (Fig. 1A). No significant fuchsin staining deposition or rete peg epithelial extensions were evident on periodic acid methenamine silver + Masson staining (Fig. 1B). This pathology had not been previously encountered at our hospital; literature consultation of the results for light microscopy indicated CG (2). Subsequent IHC examination suggested deposition of collagen III in subendothelial and mesangial regions (Fig. 2), which was confirmed by electron microscopy (Fig. 3) upon pathological diagnosis of CG. The treatment of the patient with telmisartan $(160 \mathrm{mg} / \mathrm{d}$; qd) was then initiated, which substantially relieved the clinical symptoms after half a year. Within 3 years of follow-up, the patient had only two episodes of lower-limb edema and renal function remained normal despite persistent proteinuria in regular follow-ups (Fig. 4). The plasma albumin levels remained at $\sim 30 \mathrm{~g} / \mathrm{l}$ and uric acid remained persistently slightly elevated.

\section{Discussion}

$\mathrm{CG}$, also known as collagenofibrotic glomerulopathy, is a rare idiopathic glomerular disease with massive deposition of abnormal type III collagen protein fibrils in the glomeruli (12). Persistent massive proteinuria or NS are among the first complaints in numerous patients with $\mathrm{CG}$, which then
Table I. Laboratory data of the patient at initial presentation.

\begin{tabular}{lcc}
\hline Parameter & Value & Normal range \\
\hline White blood cells $\left(\mathrm{x} 10^{9} / \mathrm{l}\right)$ & 9.0 & $3.5-9.5$ \\
Hemoglobin $(\mathrm{g} / \mathrm{l})$ & 120 & $115-150$ \\
Platelet count $\left(\mathrm{x} 10^{9} / \mathrm{l}\right)$ & 242 & $125-350$ \\
Creatinine $(\mu \mathrm{mol} / \mathrm{l})$ & 102 & $57-111$ \\
24-h urinary protein $(\mathrm{g})$ & 5.44 & $<0.15$ \\
Triglyceride $(\mathrm{mmol} / \mathrm{l})$ & 2.64 & $0.56-1.71$ \\
Cholesterol $(\mathrm{mmol} / \mathrm{l})$ & 6.81 & $2.90-5.17$ \\
Albumin $(\mathrm{g} / \mathrm{l})$ & 27.3 & $40.0-55.0$ \\
HBsAg & Negative & $0.00-0.05$ \\
Anti-HBs & Negative & $0.00-10.00$ \\
Anti-HBc & Negative & $0.00-1.00$ \\
Rheumatoid factor $(\mathrm{IU} / \mathrm{ml})$ & $<20.0$ & $<20.0$ \\
Complement C4 $(\mathrm{mg} / \mathrm{dl})$ & 21.2 & $16-38$ \\
Complement C3 (mg/dl) & 99.5 & $79-152$ \\
ANA & Negative & $<1: 100$ \\
HIV & Negative & $0-1.000$ \\
Proteinuria & $3+$ & Negative \\
IgG $(\mathrm{g} / \mathrm{l})$ & 5.25 & $7.51-15.60$ \\
IgM $(\mathrm{g} / \mathrm{l})$ & 0.787 & $0.46-3.04$ \\
IgA $(\mathrm{g} / \mathrm{l})$ & 1.68 & $0.82-4.53$ \\
ESR $(\mathrm{mm})$ & 89 & $<20$ \\
ANCA $(\mathrm{RU} / \mathrm{ml})$ & Negative & $0-20$ \\
\hline
\end{tabular}

HBsAg, hepatitis B surface antigen; anti-HBs, hepatitis B surface antibody; anti-HBc, hepatitis B core antibody; C4/3, complement component 4/3; ANA, anti-nuclear antibody; HIV, human immunodeficiency virus; Ig, immunoglobulin; ESR, erythroid sedimentation rate; ANCA, antineutrophil cytoplasmic antibody.

progress to renal damage (6). Type III collagen is normally expressed in the interstitium and blood vessels throughout the body; however, it is undetectable in glomeruli. The dysregulated formation of type III collagen is deposited in the GBM, subendothelium or mesangium, thus propagating fibrosis. According to the anatomic region of deposition, $\mathrm{CG}$ is divided into collagenofibrotic glomerulopathy and NPS (2). Deposition is mostly detected in the subendothelium and mesangium of patients with $\mathrm{CG}$, while deposition is mainly located at the basement membrane with extra-renal manifestations in patients with NPS (2). The incidence of CG is low with only 100 cases reported worldwide to date (Table II) most of them in Asia (2,13-16). To the best of our knowledge, the patient reported in the present study was the first case of CG reported in northeastern China and to be diagnosed by IHC test and further confirmed by electron microscopy.

The clinical onset of GC typically occurs between the ages of 30-50, although, recently, a case of a 3-year old female was reported (17) and there are occasional cases in older children (18). Massive proteinuria and NS are the major clinical manifestations; while certain patients have microscopic hematuria, about half of the patients present with hypertension only (without extra-renal symptoms) and typically 

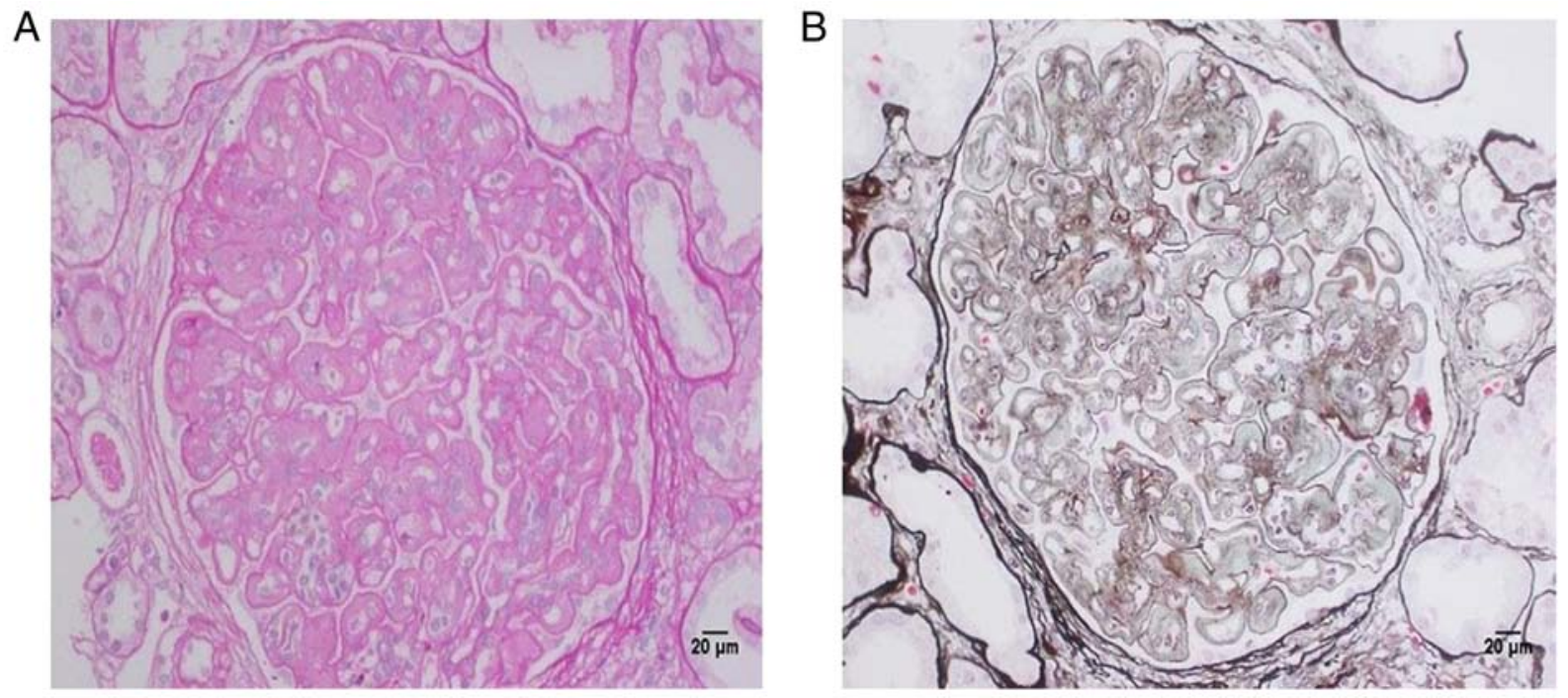

Figure 1. Light microscopy images of the biopsy specimen. (A) Section with periodic acid Schiff staining indicates mesangial expansion with positively stained material. (B) Positive periodic acid methenamine silver and Masson staining is present in certain capillary rings and there are duplications of the basal membrane (magnification, $\mathrm{x} 400$; scale bar, $20 \mu \mathrm{m}$ ).

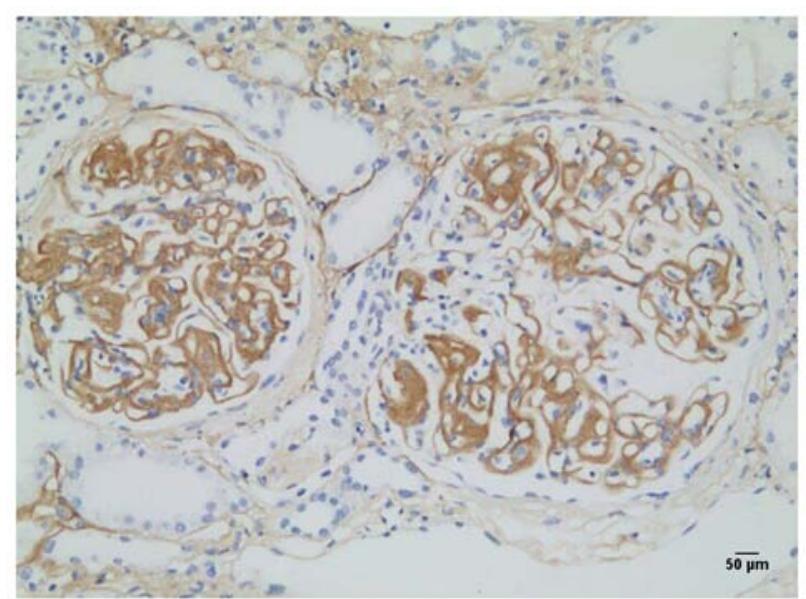

Figure 2. Immunohistochemistry images for the detection of collagen type III. A strongly positive reaction was present for collagen type III within the basal membrane. Brown staining indicates staining of collagen type III. Blue staining indicates staining of cell nuclei (magnification, x200; scale bar, $50 \mu \mathrm{m})$.

progress to end-stage renal disease $(6,19)$. There is no specific indication of $\mathrm{CG}$ in laboratory examinations, although elevated levels of the precursor of procollagen type III in the circulation may be of a certain, albeit nonspecific, diagnostic significance (3). Therefore, obtaining a correct diagnosis may be challenging.

Renal biopsy is the only definitive diagnostic method for CG and electron microscopy of biopsy material is the gold standard (2). However, electron microscopy is not available in a routine setting; therefore, IHC represents a good alternative for making a positive diagnosis (15). Previous light microscopy studies indicated mesangial expansion with weakly PAS-positive material and negative PAM staining, as well as negative IF results $(3,20)$. Electron microscopy indicates typical curved and scattered collagen fibrils in the subendothelial and mesangial regions with a periodicity of

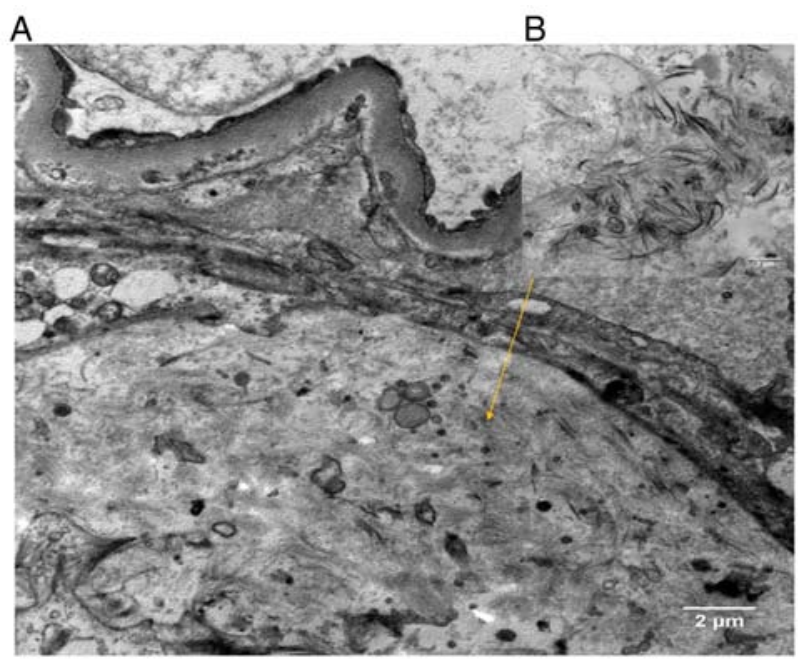

Figure 3. Electron microscopy images. (A) Electron micrograph indicates expansion of the glomerular mesangium and subendothelial space by banded fibrillar material (arrow; magnification, x12,000; scale bar, $2 \mu \mathrm{m}$ ). (B) Magnified window from A (magnification, $\mathrm{x} 30,000$; scare bar, $1 \mu \mathrm{m}$ ).

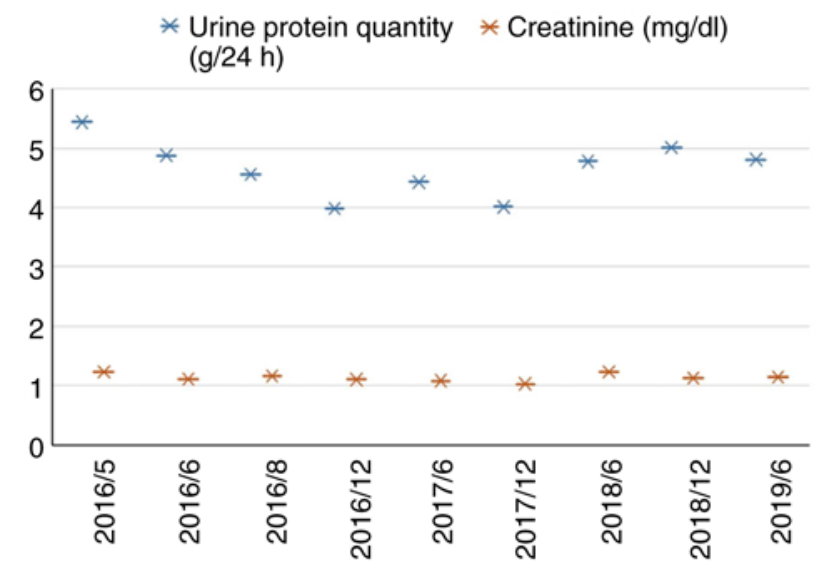

Figure 4. Creatinine levels and 24-h urine protein quantity for the patient during the 3-year follow-up. 
Table II. Cases of collagenofibrotic glomerulopathy reported in previous studies published in the English language to date.

\begin{tabular}{|c|c|c|c|c|c|}
\hline Author, year & Region & Cases (n) & Treatment $(\mathrm{n} / \mathrm{N})$ & Treatment $(\mathrm{n} / \mathrm{N})$ & (Refs.) \\
\hline Ng et al, 2020; Chen et al, 2017 & China & 33 & $1 / 33$ & $1 / 33$ & $(13,15)$ \\
\hline $\begin{array}{l}\text { Anitha et al, 2016; } \mathrm{Ng} \text { et al, 2020; } \\
\text { Matthai et al, 2020; Kurien et al, } 2015\end{array}$ & Other Asia & 57 & $3 / 57$ & $3 / 57$ & $(2,13,14,16)$ \\
\hline $\mathrm{Ng}$ et al, 2020 & North/South America & 6 & - & - & (13) \\
\hline $\mathrm{Ng}$ et al, 2020 & Europe & 2 & - & - & (13) \\
\hline $\mathrm{Ng}$ et al, 2020 & Not specified & 1 & - & - & (13) \\
\hline Total & & 99 & & & \\
\hline
\end{tabular}

,- not reported.

50-60 nm (21). IHC testing indicates strong expression of type III collagen within the mesangium and along GBMs (13). Despite these markers, differential diagnosis of $\mathrm{CG}$ from other collaged III deposition diseases, including fibrillary glomerulopathy, immunotactoid glomerulopathy and fibronectin glomerulopathy, can be distinguished by examination with an electron microscope $(15,20)$.

Although the etiopathogenesis of $\mathrm{CG}$ remains elusive, it appears that genetic factors serve an important role. An association between inherited deficiency of complement factor $\mathrm{H}$ and $\mathrm{CG}$, such that the absence of factor $\mathrm{H}$ may promote the deposition of type III collagen, has been reported (22). Furthermore, familial lineages with $\mathrm{CG}$ have been reported (23), implying that genetics may at times contribute to pathogenic pathways. However, the molecular and genetic mechanisms of the pathology of CG remain elusive and the typical adult onset may argue against a common hereditary factor. However, there remains no specific therapy for CG (24) and patients have poor prognosis, generally progressing to kidney failure within a number of years.

Inhibitors of the renin-angiotensin-aldosterone system are the first-line clinical treatment, aiming to reduce blood pressure and urine protein. Medicinal approaches include inhibitors of angiotensin-converting enzyme (ACE) and of angiotensin II receptor blockers (ARB) (25). There are certain common adverse effects of ACE inhibitors, including coughing, renal dysfunction and hyperkalemia, and, in particular, prominent angioedema (11). ARB drugs are effective in reducing the risk of end-stage renal disease in patients with diabetic nephritis and decreased serum creatinine levels two-fold compared to those obtained with ACE inhibitors (25). Therefore, due to the potent renoprotective effects and more favorable side effect profile, ARB medication was selected to treat the patient of the present study. Telmisartan was chosen because it is reportedly more effective than other ARB drugs in alleviating proteinuria (26-28). Furthermore, telmisartan is able to attenuate renal podocyte apoptosis and autophagy-associated protein expression levels (7). Finally, telmisartan may have anti-fibrotic effects in human mesangial cells and reportedly decreased the expression levels of PPAR $\gamma$ and nNOS to delay the progression of renal failure (8-11). Long term follow-up of the present case included the thorough and persistent monitoring for disease progression, which may be recommended for telmisartan as a first-line treatment for CG.
In conclusion, although most cases of $\mathrm{CG}$ have been reported in Asia, to the best of our knowledge, the present study is the first to report on a case of this very rare idiopathic renal disease in northeastern China. The present study contributes to a better awareness of CG as a rare cause of proteinuria and presents telmisartan as an effective treatment against the progression of the disease and major symptoms over a follow-up of three years.

\section{Acknowledgements}

Not applicable.

\section{Funding}

No funding was received.

\section{Availability of data and materials}

The datasets used and/or analyzed during the current study are available from the corresponding author on reasonable request.

\section{Authors' contributions}

QG and PL designed the current study and revised the manuscript. QG wrote the manuscript. LL and PN collected data. All authors read and approved the final manuscript.

\section{Ethics approval and consent to participate}

The present study did not involve any animal studies or human experimentation. The project was approved by the Medical Ethics Committee of the Second Hospital of Jilin University (Changchun, China; approval no. 2018198).

\section{Patient consent for publication}

Written informed consent was obtained from the patient and the patient allowed his clinical and pathological data to be published.

\section{Competing interests}

The authors declare that they have no competing interests. 


\section{References}

1. Dong J, Wei H, Han M, Guan Y, Wu Y and Li H: Collagen type III glomerulopathy: A case report and review of 20 cases. Exp Ther Med 10: 1445-1449, 2015.

2. Anitha A, Vankalakunti M, Siddini V, Babu K, Bonu R and Ballal S: Type III collagen disorders: A case report and review of literature. Indian J Pathol Microbiol 59: 75-77, 2016.

3. Fogo AB, Lusco MA, Najafian B and Alpers CE: AJKD atlas of renal pathology: Type III collagen glomerulopathy. Am J Kidney Dis 69: e25-e26, 2017.

4. Patro KC, Jha R, Sahay M and Swarnalatha G: Collagenofibrotic glomerulopathy-case report with review of literature. Indian J Nephrol 21: 52-55, 2011 .

5. Rørtveit R, Eggertsdóttir AV, Thomassen R, Lingaas F and Jansen JH: A clinical study of canine collagen type III glomerulopathy. BMC Vet Res 9: 218, 2013.

6. Liu H, Chen J, Zhang Y, Wang S and Zou W: Clinicopathologic features of collagen III glomerulopathy. Zhonghua Bing Li Xue Za Zhi 43: 732-735, 2014 (In Chinese).

7. Malik S, Suchal K, Gamad N, Dinda AK, Arya DS and Bhatia J: Telmisartan ameliorates cisplatin-induced nephrotoxicity by inhibiting MAPK mediated inflammation and apoptosis Eur J Pharmacol 748: 54-60, 2015.

8. Kwon YJ, Suh GH, Kang SS and Kim HJ: Successful management of proteinuria and systemic hypertension in a dog with renal cell carcinoma with surgery, telmisartan, and amlodipine. Can Vet J 59: 759-762, 2018.

9. Liang W, Chen C, Shi J, Ren Z, Hu F, van Goor H, Singhal PC and Ding G: Disparate effects of eplerenone, amlodipine and telmisartan on podocyte injury in aldosterone-infused rats. Nephrol Dial Transplant 26: 789-799, 2011.

10. Mikami D, Kimura H, Kamiyama K, Torii K, Kasuno K, Takahashi N, Yoshida H and Iwano M: Telmisartan activates endogenous peroxisome proliferator-activated receptor- $\delta$ and may have anti-fibrotic effects in human mesangial cells. Hypertens Res 37: 422-431, 2014.

11. Wang ZK, Liu ZY and Yu HB: Protective effect of telmisartan on rats with renal failure and its mechanism. Asian Pac J Trop Med 8: 498-501, 2015 .

12. Kunitomi M, Wada J, Miyatake N, Hayashi Y, Ota K and Makino H: Ultrastructure of mesangial type III collagen deposition in a patient with IgA nephropathy. Am J Kidney Dis 32: 146-152, 1998

13. Ng YF, Chow CY, Yang WS, Lye WC and Loh HL: Collagenofibrotic glomerulopathy-report of a rare renal disease with serial biopsies. Malays J Pathol 42: 131-135, 2020.

14. Matthai SM, Mohapatra A, Duhli N, David VG and Varughese S: Collagenofibrotic glomerulopathy-A rare disease diagnosed with the aid of transmission electron microscopy. Indian J Pathol Microbiol 63: S47-S49, 2020

15. Chen X, Wang H, Xu W and Zhu J: Collagen type III glomerulopathy: Case report and review of the literature. Clin Nephrol 87: 39-46, 2017.
16. Kurien AA, Larsen CP and Cossey LN: Collagenofibrotic glomerulopathy. Clin Kidney J 8: 543-547, 2015.

17. Pizzo HP, Haas M and Puliyanda D: Collagen type III glomerulopathy. Kidney Int 93: 1490, 2018

18. Alsaad KO, Edrees B, Rahim KA, Alanazi A, Ahmad M and Aloudah N: Collagenofibrotic (collagen type III) glomerulopathy in association with diabetic nephropathy. Saudi J Kidney Dis Transpl 28: 898-905, 2017.

19. Cohen AH: Collagen type III glomerulopathies. Adv Chronic Kidney Dis 19: 101-106, 2012.

20. Li L, Zou WZ, Wang SX, Wang SL, Wang W, Han ZH, Du J and Bo L: Collagen type III glomerulopathy: A morphologic study. Zhonghua Bing Li Xue Za Zhi 34: 385-388, 2005 (In Chinese).

21. Bao H, Chen H, Zhu X, Xu F, Zhu M, Zhang M, He Q, Zeng C and Liu Z: Clinical and morphological features of collagen type III glomerulopathy: A report of nine cases from a single institution. Histopathology 67: 568-576, 2015.

22. Vogt BA, Wyatt RJ, Burke BA, Simonton SC and Kashtan CE: Inherited factor $\mathrm{H}$ deficiency and collagen type III glomerulopathy. Pediatr Nephrol 9: 11-15, 1995.

23. Chen N, Pan X, Xu Y, Wang Z, Shi H, Yan F and Dong X: Two brothers in one Chinese family with collagen type III glomerulopathy. Am J Kidney Dis 50: 1037-1042, 2007.

24. Nimmagadda S, Mukku K, Devaraju SR and Uppin MS: Unusual cause of glomerular deposition disease: Collagenofibrotic glomerulopathy. Indian J Nephrol 27: 62-65, 2017.

25. Wang K, Hu J, Luo T, Wang Y, Yang S, Qing H, Cheng Q and Li Q: Effects of angiotensin-converting enzyme inhibitors and angiotensin II receptor blockers on all-cause mortality and renal outcomes in patients with diabetes and albuminuria: A systematic review and meta-analysis. Kidney Blood Press Res 43: 768-779, 2018.

26. Zheng Z, Lin S and Shi H: A systematic review and metaanalysis of telmisartan versus valsartan in the management of essential hypertension. J Clin Hypertens (Greenwich) 12: 414-421, 2010.

27. Bakris G, Burgess E, Weir M, Davidai G, Koval S; AMADEO Study Investigators: Telmisartan is more effective than losartan in reducing proteinuria in patients with diabetic nephropathy. Kidney Int 74: 364-369, 2008.

28. Naruse M, Koike Y, Kamei N, Sakamoto R, Yambe Y and Arimitsu M: Effects of azilsartan compared with telmisartan on insulin resistance in patients with essential hypertension and type 2 diabetes mellitus: An open-label, randomized clinical trial. PLoS One 14: e0214727, 2019.

(c) (1) () This work is licensed under a Creative Commons Attribution-NonCommercial-NoDerivatives 4.0 International (CC BY-NC-ND 4.0) License. 